\title{
A quelles conditions le conseil de développement peut-il faciliter le renouvellement de la démocratie locale?
}

\section{Gilles-Laurent Rayssac}

\section{(2) OpenEdition}

\section{Journals}

\section{Édition électronique}

URL : http://journals.openedition.org/developpementdurable/1004

DOI : 10.4000/developpementdurable.1004

ISSN : $1772-9971$

Éditeur

Association DD\&T

\section{Référence électronique}

Gilles-Laurent Rayssac, «A quelles conditions le conseil de développement peut-il faciliter le renouvellement de la démocratie locale? », Développement durable et territoires [En ligne], Points de vue (2003-2010), mis en ligne le 01 novembre 2003, consulté le 19 avril 2019. URL : http://

journals.openedition.org/developpementdurable/1004; DOI : 10.4000/developpementdurable.1004

Ce document a été généré automatiquement le 19 avril 2019.

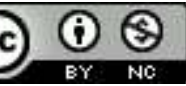

Développement Durable et Territoires est mis à disposition selon les termes de la licence Creative Commons Attribution - Pas d'Utilisation Commerciale 4.0 International. 


\title{
A quelles conditions le conseil de développement peut-il faciliter le renouvellement de la démocratie locale?
}

\author{
Gilles-Laurent Rayssac
}

Introduction

1 La réflexion sur la gouvernance locale et le développement durable offre une occasion d'aborder pratiquement la question de la démocratie. La tendance de fond que l'on constate ces dernières années conduit plutôt au pessimisme : la participation des citoyens à la vie démocratique va décroissant ; l'indicateur le plus global et sans doute le plus pertinent est le taux de participation aux élections, qu'elles soient générales ou locales: dans toutes les démocraties, il tend à diminuer. Cela doit conduire à s'interroger sur les raisons de cette défection. L'œuvre philosophique et politique de Jürgen Habermas peut nous aider à la fois à comprendre ce qui se passe et à imaginer des solutions qui, si elles n'ont pas encore été testées au niveau général, ont été expérimentées à plusieurs reprises au niveau local.

2 C'est une réflexion sur ces expériences que l'on propose de délivrer ici. Le point de départ de notre cheminement rappelle, de façon très succincte, quelques points saillants de la situation actuelle et de quelques raisons historiques qui peuvent l'expliquer (qui, du moins, y ont conduit) ; on éclairera cette situation à la lumière de l'analyse de l'espace public élaborée par Habermas et nous retrouverons une note d'optimisme en montrant en quoi le développement durable peut être l'occasion de sortir de l'ornière.

Cependant, cela ne suffit pas. On essayera de montrer que si, depuis plusieurs années, on cherche au travers de la démocratie de proximité et de la démocratie participative à élargir la participation (peut-être pour élargir le cercle de ceux qui assument des responsabilités), le conseil de développement et éventuellement les nouveaux conseils de quartier permettent de réunir les conditions nécessaires et suffisantes pour recréer un espace public de délibération propre à revivifier réellement la pratique démocratique. 
L'une de ces conditions consiste à disposer d'une procédure d'organisation de la discussion.

4 La méthode du débat public, que nous avons expérimentée à plusieurs reprises, peut être considérée comme une bonne procédure. Elle devrait permettre de modifier en profondeur l'organisation locale des pouvoirs : en ce sens, l'existence d'un conseil de développement qui fonctionne selon la méthode du débat public serait certainement l'un des meilleurs moyens de modifier et de renouveler la gouvernance des territoires.

1. L'universalisation de la démocratie et l'effondrement des corps sociaux intermédiaires nécessitent un réinvestissement dans le territoire.

5 La démocratie représentative ne suscite plus l'enthousiasme des citoyens au point que l'on explique le résultat du 21 avril 2002 par un phénomène de ras-le-bol dont les élus seraient essentiellement responsables. De son coté, la démocratie participative bégaye, n'arrive pas à s'imposer et, quand elle fonctionne, provoque frustrations, amertume et parfois violence (Gayerie, 2003). La démocratie contient en elle ses propres ferments de contestation et de contradiction ; donner la parole au peuple, c'est prendre le risque d'entendre, celui qui la prend, contester jusqu'au fait même qu'il puisse la rendre. Il n'y a sans doute pas lieu de s'émouvoir que la démocratie soit bruyante : dans ce domaine, c'est plutôt le silence qui n'est pas un bon signe.

6 Depuis les premières expériences qui ont marqué ses débuts, la démocratie est une méthode plus qu'une fin qui doit permettre à la société qui l'adopte de délibérer des meilleures solutions qui organisent la vie de tous pour que chacun puisse s'épanouir dans la liberté la plus grande. Les débuts de la démocratie ont permis, sur l'agora grecque, aux citoyens, c'est-à-dire à ceux qui n'avaient pas besoin de travailler pour vivre, d'expérimenter cette forme d'organisation sociale: ils étaient un petit nombre, se connaissaient tous et pratiquaient cette «discipline» dans un espace réduit sur lequel les règles étaient facilement lisibles et tout aussi facilement adaptables en cas de nécessité. Les différentes expériences démocratiques qui ont été vécues au cours de l'histoire des 2300 années qui ont suivi ont toutes été plus ou moins réalisées dans des conditions similaires: espace géographique réduit, nombre de "personnes concernées» (les citoyens) peu important, temps largement disponible des citoyens. C'est avec l'avènement de la démocratie américaine que les conditions de la vie démocratique sont entrées dans leur phase moderne qui se caractérise par une généralisation (au moins à des aires géographiques) puis à une universalisation (loin d'être atteinte, encore) de cette méthode d'organisation sociale. C'est le mouvement même de généralisation - universalisation qui donne à la démocratie l'apparence d'une fin sapant ainsi ses propres bases (Gauchet, 2002). En cherchant à rendre démocratique l'ensemble des parties du monde comme l'ensemble des organisations sociales, on finit par croire que l'état démocratique est l'objectif. Pourtant, on sait bien que la démocratie n'est pas un état mais un processus: «les sociétés démocratiques [sont] celles où les conditions du vivre ensemble ne sont pas définies a priori, fixées par une tradition ou imposées par une autorité» (Rosanvallon, 2003, p.14).

$7 \mathrm{Au}$ cours des cent cinquante années qui ont suivi la «création» de la démocratie américaine et avec l'élan donné, dans le fracas, par la Révolution française, cette forme d'organisation a fait nombre d'émules; mais, jusqu'à une période finalement récente, la démocratie reposait sur un système d'organisation sociale assez fortement structuré: les partis politiques, les organisations syndicales, les mouvements de jeunesse eux-mêmes rattachés aux partis ou aux églises, les mouvements d'éducation populaires étaient autant 
de piliers sur lesquels reposait la pratique démocratique, autant d'écoles qui faisaient œuvre de pédagogie pour les jeunes, mais aussi pour les moins jeunes qui, en permanence, étaient conduits à refaire leurs gammes sur les règles démocratiques. Cette structuration avait deux caractéristiques importantes: d'une part elle donnait une homogénéité sociale qui, sans constituer des «classes sociales», organisait les groupes en gommant en partie les différences territoriales; d'autre part elle apportait à tout un chacun une méthode qui, peu ou prou, était partout la même et reposait sur la logique de la représentativité; au niveau des organisations sociales comme au niveau de la République, la règle était la même pour tous: un homme, une voix, les mandants représentés par des élus représentatifs, les décisions prises à la majorité et s'imposant à tous.

Depuis, plusieurs événements sont venus modifier considérablement les conditions d'exercice de la démocratie en France : les corps sociaux intermédiaires ont une influence beaucoup moins importantes qu'auparavant, voire nulle dans de nombreux cas ${ }^{1}$, notamment parce que les idées et doctrines qu'ils produisaient (et produisent encore) n'ont plus de médias indépendants (ou dépendants : tout dépend le point de vue que l'on adopte) qui les véhiculent ; les médias actuels opèrent une fonction de filtre qui affaiblit considérablement la portée du discours de ces corps intermédiaires ${ }^{2}$. D'autre part, la construction de l'Europe qui met en cause la puissance de l'Etat-nation, et surtout la mondialisation qui fait éclater tous les schémas traditionnels d'explication du monde, rendent plus nécessaire l'attachement à un territoire, dans un réflexe de recherche ou d'affirmation identitaire et de citoyenneté revendiquée: «La problématique du territoire devient cruciale, comme le prouvent, notamment en Europe, les différents foyers de conflits [...] Plus on vante les vertus de la communication, plus en contrepoint, émerge le besoin "d'être de quelque part". Ce profond mouvement de balancier anthropologique concerne également la politique. Le grand changement ici est la mondialisation des enjeux. Tout devient mondial, mais si l'on ne donne pas aux citoyens les moyens d'agir et de se réaliser au plan local, le déséquilibre sera catastrophique. Le local est le symétrique indispensable à l'élargissement constant des frontières» (Wolton, 19963).

9 Ainsi, l'évolution des dernières décennies conduit-elle à la situation présente dans laquelle l'existence d'un cadre démocratique global (la Constitution et les pratiques générales du monde politique et administratif) reste incontestable; cependant, ce cadre est de moins en moins rattaché à une pratique concrète de la vie démocratique : les électeurs sont des intermittents de la démocratie qui ont pour seule action de déposer un bulletin dans l'urne électorale à chaque scrutin. Et cela, alors même que l'investissement dans l'identité territoriale est de plus en plus nécessaire pour reconstituer un système de repères qui permettent de mieux se situer dans un monde globalisé.

2. L'espace public s'est atomisé rendant plus difficile la définition de l'intérêt général.

10 L'analyse de l'espace public, développée par Jürgen Habermas, même si elle porte sur une période historique différente, permet de mieux comprendre la situation actuelle, les dysfonctionnements de la vie (de la pratique) démocratique et aide à fixer un cadre d'analyse des tentatives actuelles de revivification de la participation des citoyens à la vie publique. Pour J. Habermas, l'espace public est un « réseau permettant de communiquer des contenus et des prises de position, et donc des opinions ; les flux de la communication y sont filtrés et synthétisés de façon à se condenser en opinions publiques regroupées en fonction d'un thème spécifique » (Habermas, 1997, p. 387). 
11 C'est le lieu où les opinions et les aspirations se confrontent pour, à la fois, construire l'intérêt général et établir une distance critique vis-à-vis de l'action de l'Etat. Habermas a forgé sa propre analyse de l'espace public dans l'étude de ce qu'il appelle l'espace public bourgeois qui s'est constitué au XVIIIème siècle, en réaction à un Etat totalitaire : la Publicité (c'est-à-dire le fait de rendre publique l'information d'Etat) était le moteur de cet espace public bourgeois qui a correspondu, un temps, avec des aspirations universelles notamment au moment de la première période révolutionnaire en France. Cependant, cet espace public s'est rapidement consolidé par la répression de l'opinion publique plébéienne en détournant le rôle de la Publicité ; au moment où Habermas l'analyse, c'est-à-dire dans les années 50 , il considère que cet espace public n'arrive plus à rendre compte de l'espace politique de la démocratie de masse. Ainsi, «depuis un siècle environ, ses fondements sociaux sont sans le moindre doute à nouveau en voie de disparition; on ne peut ignorer certaines tendances qui vouent [la sphère publique] à sa perte: tandis que l'aire qu'elle couvre ne cesse de s'étendre, l'importance de son rôle décroît sans discontinuer». L'analyse de Habermas, très pessimiste dans sa première version, plus optimiste à la suite de sa préface de 1990, reste valable pour la période actuelle; du moins peut-elle nous servir de modèle d'analyse de la situation qui prévaut aujourd'hui. En effet, l'espace public aujourd'hui se caractérise par une double apparence: d'un coté, il est strictement réduit à ce que l'on pourrait nommer la «sphère politique pure» (et que d'aucun ont appelé, mi-ironique, mi-méprisant, le «microcosme») dans laquelle entrent en jeu essentiellement les élus (au moins par fonction : tous n'ont certainement pas la maîtrise de l'ensemble du processus qu'ils sont censés piloter), les mass média et les technocrates ; eux seuls sont à même de donner l'apparence (ou l'illusion) de maîtriser la complexité de ce que l'on peut appeler la vie quotidienne collective, c'est-à-dire de l'ensemble des processus de recherche de l'intérêt général organisés de telle sorte qu'ils ne débouchent pas sur une paralysie du système ou une révolte d'une catégorie qui s'estimerait mal traitée ou négligée ; d'un autre coté, l'espace public semble atomisé en de multiples espaces autonomes, mi-publics, mi-privés, qui sont constitués de groupes plus ou moins importants qui tentent (ou non) d'avoir une influence sur la "sphère politique pure ", mais qui ont tous pour fonction de permettre de construire une représentation thématisée d'un espace public à dimension variable. Ainsi, les altermondialistes forment-il un " espace public » aussi sûrement que les défenseurs des oiseaux migrateurs. Si cette atomisation est aujourd'hui possible et sans doute inéluctable et irréversible, c'est parce que l'espace public tel qu'il a été étudié par Habermas ne peut plus être considéré comme un tout: il a cédé sous la pression et les coups de boutoir des mass média et le développement sans précédent d'une information généralisée, universelle, omniprésente et finalement facile d'accès parce que peu coûteuse mais, en même temps, de moins en moins maîtrisable. D'une certaine manière, alors que l'espace public analysé par Habermas avait un caractère englobant qui faisait de cet espace un tout dans lequel s'organisait la vie collective (lieu à la fois du politique - au sens que Rosanvallon donne à ce terme (Rosanvallon, 2003) - de la vie sociale et de la société civile), aujourd'hui, ce «tout», cette totalité n'existe plus (Habermas, 1962 - 1993, cf. la préface de 1990) et ce qui en apparence en tient lieu n'est plus à coup sûr, la sphère politique mais la sphère informationnelle: seuls les mass média donnent aujourd'hui (l'illusion d') une explication globale du monde; peu importe d'ailleurs qu'elle soit juste: il suffit pour être crédible qu'elle se déclare comme telle. La conséquence directe et immédiate de cette évolution est que l'espace politique, le lieu de la constitution de la décision publique (qui a pour première caractéristique d'engager l'argent public, celui de 
nos impôts: c'est un lien direct avec la vie quotidienne) est un espace verticalisé, construit en forme de tuyaux d'orgue, qui interdit toute représentation globale et ne permet pas de resituer chaque décision dans son ensemble logique et stratégique. D'où à la fois un désengagement des "gens ordinaires» du débat politique banalisé dans lequel ils ne se retrouvent pas (au sens premier du terme: ils n'y sont pas) et une sur-spécialisation de ceux qui s'investissent le plus dans la vie publique, dans une logique de défense d'intérêts catégoriels ou d'intérêts généraux spécialisés. Les défenseurs de la réintroduction du loup dans les Alpes agissent à n'en pas douter dans le cadre de l'intérêt général ${ }^{4}$ mais refusent généralement de prendre en considération les peurs et les malheurs des éleveurs ; lesquels, en s'opposant par la violence à cette politique, ne défendent qu'un intérêt catégoriel. Le problème du monde politique, aujourd'hui, est qu'il ne sait pas résoudre ce type de difficulté ; n'est-ce pas là un signe, parmi d'autres, du dysfonctionnement de l'espace public?

3. Diverses tentatives pour redonner du sens au politique

12 Depuis 25 ans, des tentatives de plus en plus nombreuses, traduisent la recherche de solution à la «désaffection de gens», mais il semble que les lois et décrets ne pourraient rien sans l'apparition, presque inopinée du développement durable.

13 En effet, depuis plusieurs années, deux idées semblent être utilisées comme antidote à la «désaffection des gens» pour la chose publique: il s'agit de la démocratie participative d'une part et de la démocratie de proximité d'autre part. La première repose sur l'idée qu'il est nécessaire de faire «participer» les gens à la vie publique. Cette idée est déclinée selon plusieurs modalités qui s'échelonnent entre deux extrêmes : de la participation à la décision (qui va jusqu'à mettre les élus sous la quasi-tutelle du peuple (Rosanvallon, 2000): on est alors proche d'aspirations historiques à la démocratie directe) à la participation à la vie publique en général. Dans ce cas, l'objectif de la participation n'est pas vraiment précis, il s'agit en fait d'une influence des méthodes de «management participatif» qui ont montré, dans le monde de l'entreprise, que l'implication des gens dans les processus d'organisation facilite la responsabilisation et permet de limiter les revendications. La seconde idée, celle de la démocratie de proximité repose sur un postulat et une intuition : le postulat, c'est que la démocratie est plus «vraie» si elle est plus proche (dans une logique de retour vers l'agora grecque?). Bien des exemples de potentats locaux démontrent aujourd'hui même que c'est loin d'être toujours vrai, parce que ce n'est pas la distance qui compte, mais plutôt la méthode. Mais, en revanche, une intuition semble juste: la proximité, c'est le territoire d'appartenance, c'est donc une dimension complémentaire de la recherche d'identité telle qu'elle a été évoquée plus haut.

14 C'est pour mettre en œuvre ces deux idées que les pouvoirs publics ont adopté, au cours des vingt dernières années, plusieurs dispositions législatives susceptibles de favoriser la participation des citoyens à la vie publique : des enquêtes publiques (1983) à la démocratie de proximité (27 février 2002) en passant par la loi relative à l'administration territoriale de la République (loi ATR) de 1992 ; elles sont venues compléter un dispositif déjà fourni d'instances diverses qui avaient toutes pour fonction, dans des champs donnés, de consulter des citoyens ${ }^{5}$ qui ne sont pas représentatifs des " gens ordinaires " mais qui ne sont pas non plus des élus ou des technocrates (Zémor, 2003). Les deux grandes nouveautés de la période récente sont la création des conseils de développement (loi d'orientation pour l'aménagement et le développement durable du territoire LOADDT - du 25 juin 1999) et des conseils de quartiers (loi de démocratie de proximité du 
27 février 2002); les uns et les autres ont été conçus, au plan législatif, de la même manière : une définition très générale qui laisse à l'appréciation des acteurs locaux le soin de préciser les modalités de création, de mise en œuvre et de fonctionnement de ces nouveaux outils de la «démocratie participative de proximité». Même s'il existait des expériences diversifiées de conseils de quartier depuis plusieurs années (certaines villes ont institué ces conseils dès le milieu des années soixante-dix), aujourd'hui, l'expérience qui semble être la plus prometteuse est celle des conseils de développement notamment parce que leurs «compétences» sont nettement plus large que celles des conseils de quartier. On remarquera en particulier que les conseils de développement sont directement liés aux principes du développement durable puisqu'ils sont présentés, dans la LOADDT, comme un instrument au service d'une approche de type «développement durable» de l'aménagement du territoire.

Or, même si l'on peut retrouver des prémices du développement durable jusque dans les années soixante, l'irruption quasiment inopinée ${ }^{6}$ de ce concept redonne un véritable espoir politique face au désenchantement démocratique (Perrineau, 2003), un espoir de recoller les morceaux au sein de la sphère politique ou, plus exactement, à l'articulation entre celle-ci et les différentes sphères autonomes qui se sont constituées au fil des dernières décennies. D'une certaine façon, donc, le développement durable permet et nécessite la résurgence d'un espace public qui s'était trouvé émietté.

Le développement durable peut être compris comme un concept opératoire plutôt que comme un concept normatif : en ce sens, le développement durable n'est pas une politique au sens où le développement économique peut être l'objet de diverses stratégies politiques, plus ou moins interventionnistes, plus ou moins libérales, dotées d'instruments, de dispositifs voire d'institutions et de services qui en assurent la mise en œuvre quotidienne. $\mathrm{Si}$, pour respecter une démarche de développement durable, il est utile de fixer des objectifs (y compris quantitatifs, donc des normes) comme par exemple dans le domaine de l'environnement ou dans celui de la parité, ce qui semble le plus prometteur dans le développement durable, c'est bien sa dimension opératoire et méthodologique : s'il dit ce qu'il faut faire, il dit aussi (et surtout) comment le faire aujourd'hui de façon à ce qu'on puisse continuer de le faire demain. Cette caractéristique du développement durable constitue sa force mais, elle est, en même temps, source de la complexité qui rend le terme difficile à appréhender tant au plan conceptuel qu'au plan pratique.

éveloppement durable, comme son nom l'indique, est une approche qui refuse le non-

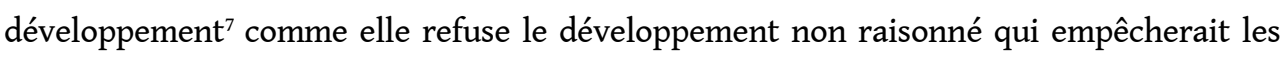
développements futurs; il s'agit d'une méthode nouvelle de l'organisation des hommes et des femmes et de leurs projets. En ce sens, le développement durable n'est évidemment pas sans rapport avec la question de la construction démocratique. De par sa portée et son ambition (plus exactement, l'ambition et les espoirs que l'on place en lui), le développement durable réintroduit du débat politique ; il a bien pour finalité principale de rechercher la conciliation des intérêts souvent divergents des parties prenantes.

On peut ainsi mettre en avant trois caractéristiques majeures du développement durable qui forment la matrice d'une grille de lecture du concept et de ses mises en pratiques. D'une part, en reliant le présent et le temps futur, en solidarisant ce qui peut être fait aujourd'hui et ce qui doit pouvoir être fait demain, le développement durable est un indicateur incontestable de ce qu'est l'intérêt général. En second lieu, en préconisant une approche globale et intégrée, en rendant indispensable une vision systémique, le 
développement durable est une clef d'accès à la maitrise de la complexité du monde dans lequel nous vivons. Enfin, en troisième lieu, en étant à la fois un indicateur de l'intérêt général et une clef d'accès à la maitrise de la complexité, le développement durable embrasse solidairement toutes les activités humaines individuelles et collectives, publiques et privées.

4. Quatre conditions sont nécessaires pour que le conseil de développement soit le lieu de la délibération du développement durable

"Composé de représentants des milieux économiques, sociaux, culturels et associatifs (...) le conseil de développement s'organise librement. Il est associé à l'élaboration de la charte de pays. Il peut être consulté sur toute question relative à l'aménagement et au développement du pays. Le conseil de développement est informé au moins une fois par an de l'avancement des actions engagées par les maîtres d'ouvrage pour la mise en œuvre du projet de développement du pays et est associé à l'évaluation de la portée de ces actions ${ }^{8} »$.

Première condition: le conseil de développement n'est pas une «institution» classique, mais une innovation qui peut changer considérablement la manière de travailler des élus. C'est la raison pour laquelle il faut que les élus, ceux qui ont le pouvoir de décider en dernier ressort, soient disposés à laisser vivre un espace de délibération qui aura aussi pour fonction de les interroger, de les interpeller, de leur demander des comptes. Il faut qu'une approche de type développement durable soit clairement et volontairement adoptée par les responsables (politiques mais aussi administratifs) du territoire.

Seconde condition: il est nécessaire que la composition du conseil de développement autorise effectivement la confrontation de points de vue différents relatant des expériences différentes de la vie quotidienne; il ne s'agit pas ici de chercher nécessairement une représentativité de la population car, le conseil de développement ne relève pas de la logique représentative. En revanche, pour que cette instance joue pleinement son rôle qui consiste à éclairer la décision publique en thématisant la vie quotidienne, c'est-à-dire en permettant que les éléments qui constituent la multitude des petits et grands espoirs, des tracas et des plaisirs «des gens» se transforment, par une sorte de cristallisation due au processus de discussion, en volontés collectives, en opinions puis en choix politiques. Pour cela, la composition du conseil de développement doit permettre à plusieurs types de contribution d'être portés au débat: le témoignage, l'information, l'expertise technique, la vision politique, etc.

De ce fait, et c'est la troisième condition, le conseil de développement, pour remplir son office, doit être conçu dans une logique systématique ; cette approche, globale et intégrée, est la seule qui permettra que se réalise le processus de cristallisation propre à une réelle thématisation de la vie quotidienne. Il doit permettre en effet de passer d'une approche verticale, sectorisée et fonctionnelle des choses à une vision non seulement horizontale ou transversale, mais encore une vision dans laquelle on fait ressortir les éléments clefs, ceux qui font levier, les nœuds à partir desquels l'action présente plusieurs dimensions. Par exemple, on peut analyser de plusieurs façons, sur un territoire, la question des transports collectifs, celle de la répartition des équipements collectifs (les installations sportives, par exemple), les horaires d'ouverture des magasins et des écoles et celle des moyens que se donne la collectivité pour favoriser l'éveil et l'éducation des enfants en dehors des horaires scolaires. Ces questions peuvent être analysées, et c'est ainsi que cela se passe le plus souvent, séparément les unes des autres ; elles peuvent aussi, c'est plus rare, être prises en compte de façon simultanée ; il n'en 
reste pas moins que ces questions, dans la vie quotidienne, vont se concrétiser sous la forme du casse-tête hebdomadaire de la mère de famille qui doit, dans le même temps, conduire son fils de 8 ans au foot, sa fille de 12 ans à l'escrime, aller faire les courses au supermarché, rentrer chez elle préparer le repas du soir et retourner chercher ses enfants en fin d'après-midi'. Il y a un monde entre la manière de poser les problèmes de la vie quotidienne et la façon de les vivre. Or, le conseil de développement semble être, historiquement, le premier espace de débat qui rend possible le changement de perspectives et permet de réconcilier l'approche technique (technocratique ?) et l'approche vécue d'une même réalité sur un territoire (en cela, le conseil de développement, sous réserve de quelques conditions qui sont énoncées ci-dessous, est une invention tout à fait nouvelle).

Cela est rendu possible par le fait que, pour la première fois, le législateur a institué un lieu qui permet de mettre en commun ces différentes approches. Ce faisant, ce lieu, le conseil de développement, permet de construire une vision politique de la réalité vécue: en rendant possible la confrontation de plusieurs types d'approches, le conseil de développement opère cette thématisation évoquée par J.Habermas quand il décrit la théorie de la discussion. Il s'agit de cette possibilité offerte par la discussion d'exprimer des points de vue, d'échanger des expériences, de mettre en commun des visions diverses de la réalité vécue, parfois convergentes, parfois divergentes. C'est de ces échanges, constitués de la relation de faits de la vie quotidienne et d'opinions émises à leurs propos que procède la capacité à établir une distance critique et auto-réflexive qui permet d'aboutir à la construction d'une vision commune qui exprime un agir collectif. Ce processus de délibération a aussi été analysé par Hannah Arendt qui considère que l'homme ne peut devenir acteur de sa propre histoire qu'au travers du médium de la parole publique. Bref, on pourrait envisager le conseil de développement comme un véritable espace public, au sens habermassien du terme. Du moins peut-on faire cette hypothèse qu'au niveau d'un territoire, au niveau local, le conseil de développement offre une telle possibilité ${ }^{10}$.

Cela nous conduit à étudier la dernière condition qui permet d'envisager le conseil de développement comme un espace public de délibération. Si le conseil de développement, offre bien un lieu qui peut héberger la discussion, cela n'est pas suffisant pour garantir l'existence d'un espace public de délibération qui conduirait effectivement à la construction de véritables opinions politiques et à l'émergence d'un agir collectif. Pour que la construction soit complète, il faut qu'à l'existence du lieu s'ajoute la réalité d'une procédure. En effet, quand J. Habermas décrit l'espace public de discussion qui permet la délibération publique, il suppose que ces deux éléments sont réunis: un lieu et une procédure. C'est pourquoi d'ailleurs il fait souvent référence, dans ses différents textes, à l'espace judiciaire: le tribunal est l'exemple le plus souvent cité par J.Habermas quand il veut faire comprendre ce qu'est un espace public de délibération. Le tribunal est le lieu (consacré) dans lequel une procédure (le code de procédure pénale) règle la discussion judiciaire: les deux conditions nécessaires et suffisantes à l'existence d'un espace public de délibération (judiciaire) sont ainsi réunies.

On se permet d'y insister car la réalité est que l'absence de méthodologie efficace et performante en matière d'organisation du débat public débouche, bien souvent, sur des difficultés récurrentes rencontrées dans les différents lieux d'exercice de la démocratie participative. Il est frappant de constater que les responsables politiques qui expérimentent les différentes formules de démocratie participative (conseils de quartier, 
conseils de développement ou tout autre forme) remettent régulièrement leur ouvrage sur le métier, cherchent en permanence des formes nouvelles d'organisation, modifient très souvent le fonctionnement de ces instance ${ }^{11}$. Cela concerne ceux d'entre eux qui n'abandonnent pas à la première difficulté et se battent pour que l'expérience réussisse; mais combien de ces structures, en cherchant leur second souffle, se sont épuisées?

5. La méthode du débat public

Pour Habermas, l'espace public ne se «crée pas à volonté» (Habermas, 1997, p.392) ; en ce sens nous nous inspirons librement de ses écrits dans la mesure où il semble nécessaire, aujourd'hui, d'avoir une action volontariste pour «élargir la participation» de manière à susciter un espace public (qui permettra la formation d'une opinion publique, capable d'avoir une influence sur le politique). Au passage, on remarquera que ce souci d'élargir la participation se réfère, plus largement, à la nécessité d'accroître les pratiques démocratiques ou, plus précisément, d'augmenter le nombre d'occurrences au cours desquelles il est possible de pratiquer l'exercice démocratique. D'une certaine façon, il en va de la santé du corps social: la pratique de la démocratie est à ce dernier ce qu'est la pratique de la gymnastique au corps humain.

Cela pose la question de la procédure. J.Habermas fonde son modèle de politique délibérative qui se veut être une synthèse entre les conceptions libérales et républicaines de la politique, sur la théorie de la discussion ; celle-ci permet que se forment des opinions publiques et une volonté politique qui débouchent sur l'action. La théorie de la discussion peut fournir une méthode intéressante dans la mesure où elle autorise sur «des processus d'entente qui se déroulent, d'un côté, sous la forme institutionnalisée de délibérations menées dans les corps parlementaires et, de l'autre, dans le réseau des communications des espaces publics politique» (Habermas, 1998, p.270); elle met ainsi clairement en relation le contenu des communications de l'espace public avec le fonctionnement politique institutionnel. Pour exprimer la même chose autrement, on peut écrire que la théorie de la discussion permet d'articuler démocratie représentative et démocratie participative. Elle permet donc d'apporter une première réponse à la question de l'évolution des modalités de gouvernance puisque l'articulation évoquée ici est l'un des plus sûrs moyens de provoquer cette évolution. Cependant, nous divergerons pratiquement de l'énoncé habermassien dans la mesure où il considère que la formation de l'opinion publique est " informelle », c'est-à-dire qu'elle se forme d'elle-même, qu'elle ne peut être ni instrumentalisée, ni institutionnalisée, ni organisée. Une telle conception demande des conditions idéales qu'il semble relativement aléatoire de rencontrer dans le monde vécu. En outre, si l'on peut envisager de tels processus pour la délibération de grandes questions qui concernent l'ensemble de la société (sans que l'on sache toujours décrypter ce qui relève de la libre opinion et ce qui est le résultat de manipulations plus ou moins «volontaires» des mass média), pour la conduite d'actions de développement territorial, une procédure moins informelle, faisant appel à l'expertise, nous semble nécessaire. Cette procédure, nous l'appelons la «méthode du débat public».

La méthode du débat public, que nous avons expérimentée à plusieurs reprises sur des opérations ponctuelles ou de plus long terme, offre une procédure possible d'organisation des débats d'un conseil de développement. La «méthode du débat public» est une méthode d'intervention sur les territoires que nous avons élaborée à la suite de plusieurs années d'expérience d'évaluation des politiques publiques et de pratique du diagnostic territorial. Cette méthode est une pratique participative et endo-formative qui répond à des caractéristiques précises inspirées de la théorie de la discussion tout en empruntant 
aux méthodes d'élaboration collective. Elle répond à deux principes organisateurs : d'une part, le principe de double non-spécialisation qui impose de ne pas organiser de débat spécialisé et de ne pas organiser de débats uniquement entre spécialistes, afin de laisser ouvert le champ de la complexité et de son appréhension. D'autre part le principe de double finalité : la méthode du débat public vise à la fois à produire de la connaissance partagée et à mobiliser vers l'action.

Elle permet de mettre à jour les mécanismes relationnels au sein d'un partenariat et d'éclairer une situation donnée en faisant apparaître les zones de consensus, les zones de dissensus ${ }^{12}$ et en les gérant en tant que telles, de sorte que les décideurs puissent, en connaissance de cause, prendre les décisions qui s'imposent. Cette méthode permet de confronter des points de vue différents et d'aboutir à des consensus par apports de diverses origines, comme on l'a déjà mentionné plus haut (information construite par des spécialistes, expertises, témoignages, données brutes, etc.).

De la même manière que la théorie de la discussion offre la possibilité de faire évoluer les modalités de gouvernance en général, la méthode du débat public permet, elle, de renouveler les modalités de gouvernance des territoires. Notamment parce que la méthode du débat public permet de ré-articuler les pouvoirs. En effet, donner la parole à l'ensemble des acteurs représentant les forces vives du territoire conduit à élargir la participation et à faire bouger les lignes des différentes formes de pouvoir qui sont à l'origine du développement et des stratégies de changement (et de management) territorial. On passe d'un système relativement linéaire dans lequel les pouvoirs d'imaginer, de décider et de faire s'exercent successivement et sont organisés, pour l'essentiel, autour de l'élu (local) et de la puissance administrative ${ }^{13}$ (qui peut être, par ailleurs, la représentante de l'élu d'un autre niveau) à un système dans lequel, a minima, les pouvoirs d'imaginer et de décider sont exercés conjointement et simultanément avant de trouver leur expression concrète dans le pouvoir de faire de l'administration. Mais, on peut aussi imaginer que les pouvoirs s'articulent entre eux dans une autre logique, plus complexe encore, mais sans doute plus proche de la réalité parce qu'elle laisse à l'administration, qui exerce le pouvoir de faire, une part, bien réelle, de capacité autonome d'agir (on le voit dans le monde vécu). Dans ce cas, on passe à un schéma d'organisation qui place les trois pouvoirs l'un par rapport à l'autre dans une position d'influence réciproque même si le pouvoir de décider (apanage de l'élu) reste l'ultime pouvoir.

Schéma de l'articulation traditionnelle des trois pouvoirs sur le territoire 
Elus et élites

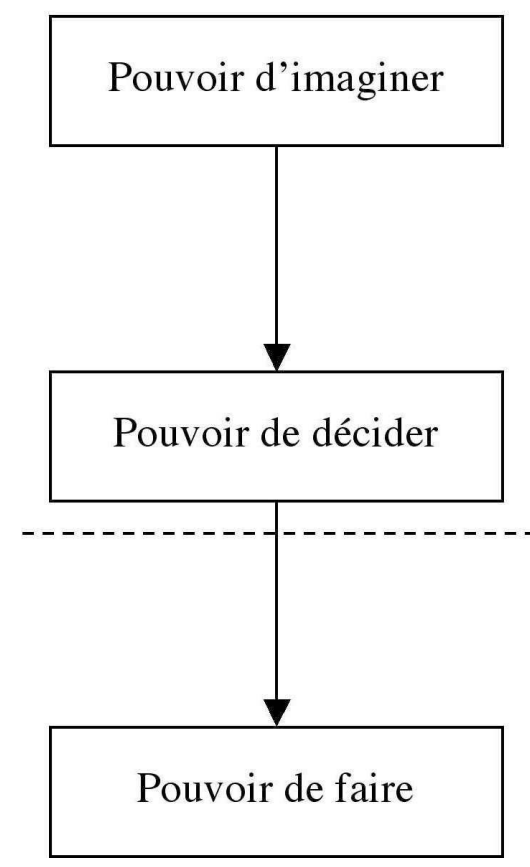

Schéma de l'articulation des trois pouvoirs après modification des modalités de gouvernance sur le territoire

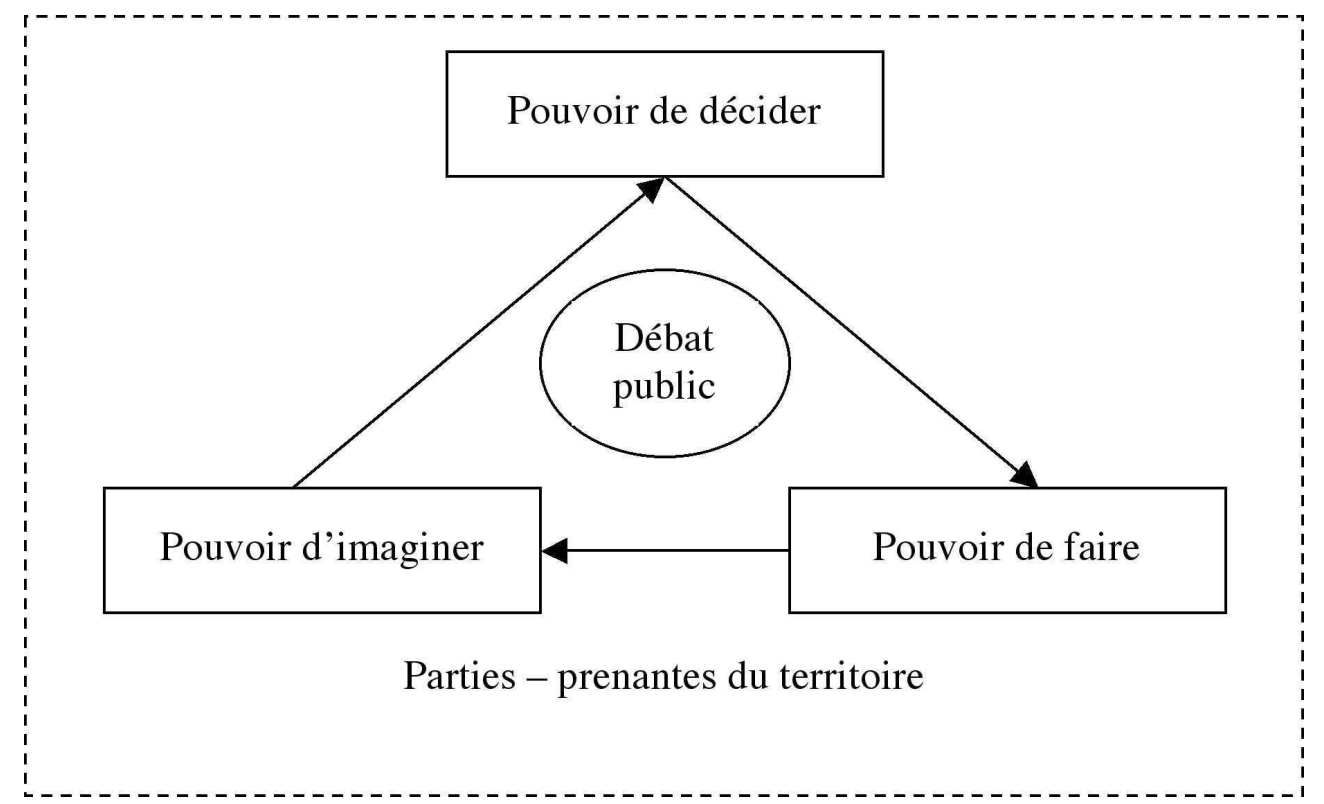

Enfin, cette ré-articulation des pouvoirs permet de mieux penser l'avenir et le territoire parce qu'en adoptant la méthode du débat public, on se situe d'emblée au niveau de la réflexion stratégique globale: en effet, en faisant participer l'ensemble des acteurs du territoire dans un processus délibératif qui reconnaît à chacun la capacité à être force d'analyse et de proposition, en ouvrant le pouvoir de créer à toutes les influences locales, on ouvre la possibilité de prendre en compte, dans un même mouvement de réflexion collective, l'ensemble des dimensions du «système territoire»: les ressources humaines, l'appareil productif, les dynamiques territoriales. 

n'est pas un faux-semblant, c'est-à-dire en s'assurant qu'il existe un espace public de délibération et une procédure (ici, la méthode du débat public) qui organisent et rendent possible la formation d'opinions publiques et de volontés politiques, on parvient à faire suffisamment bouger les lignes pour provoquer une évolution des modalités de gouvernance. Cette évolution permet l'émergence d'une capacité nouvelle à penser le territoire et son avenir, de façon à la fois stratégique et partagée. Enfin, et l'on peut espérer que cela assure une certaine pérennité à cette évolution, l'élargissement de la participation, dans cette optique, permet de ré-articuler les pouvoirs d'imaginer, de décider et de faire, ce qui revient, ipso facto, à réorganiser le fonctionnement du territoire.

faisant, on pourrait aborder des questions qui n'ont pas été vues ici, ou seulement effleurées: nous pensons en particulier à la relation absolument nécessaire mais complexe (Gaux-Baudiment, F., Heurgon, E., Landrieu, J., 2001) dans notre méthode du débat public, entre les experts et les non experts. Nous pensons aussi, par conséquent, à l'articulation des différentes formes d'apport dans le débat public: puisque celui-ci doit répondre au principe de double non-spécialisation, comment les différents apports sont-ils amalgamés pour aboutir à un consensus et à une entente suffisamment forts pour influer effectivement sur la décision politique? Dans le même ordre d'idées, se pose la question de l'articulation intime entre les trois formes de pouvoir. Un modèle serait à mettre au point pour, à partir de la méthode du débat public, éclairer cette relation à trois. Il nous semble, mais ce n'est qu'une hypothèse que nous avançons avec prudence, que cette question pourrait permettre de dépasser celle de l'articulation entre démocratie représentative et démocratie participative qui, comme l'indique P. Rosanvallon (2000), reste un chantier largement inachevé.

\section{BIBLIOGRAPHIE}

Arendt, H., 2002, Condition de l'homme moderne, Paris, Pocket, Essais.

Debray, R., 1991 et 2001, Cours de médiologie générale, Paris, Gallimard.

Debray, R.,2001, Introduction à la médiologie, Paris, Gallimard.

Gauchet, M., 2002, La démocratie contre elle-même, Paris, Tel, Gallimard.

Gayerie, J.P., 2003, «La démocratie participative dans tous ses états», La lettre du cadre territorial, $\mathrm{n}^{\circ} 253,15$ mai 2003, p.36-38.

Goux-Baudiment, F., Heurgon, E., Landrieu, J., 2001, Expertise, débat public : vers une intelligence collective, Cerisy, Edition de l'Aube.

Habermas, J., (1962)1993, L'espace public, Paris, Payot.

Habermas, J., 1986, Morale et communication, Paris, Le Cerf.

Habermas, J., 1997, Droit et démocratie, Entre faits et normes, Paris, NRF Essais, Gallimard. 
Habermas, J., 1998, «Qu'est-ce que la politique délibérative?» in L'intégration républicaine, Essais de théorie politique, Fayard, p.270

Hartereau, A., 2002, Communication publique territoriale et démocratie participative, La Lettre du cadre territorial éditeur, Dossier d'experts, $126 \mathrm{p}$.

Perrineau, P., 2003, Le désenchantement démocratique, Paris, Essai, L'aube.

Rayssac, G.L., El Fallah, M.C. et de Villeneuve, C., La démocratie participative : analyse de trente expériences territoriales, à paraître.

Rayssac, G.L. et al., 1999-2000, L'évaluation ex ante du DOCUP Objectif 22000 - 2006 en Limousin, Préfecture de la région et Conseil Régional du Limousin.

Rayssac, G.L. et al., Mission d'accompagnement de l'émergence du Pays de Cotentin, Comité de Bassin d'emploi du Cotentin, 2001-2002.

Rayssac, G.L., Mission d'accompagnement du changement territorial, bassin d'emploi de Bourges, DIRRD, Ministère de la Défense, 2002-2003.

Rosanvallon, P., 1998, Le peuple introuvable, Histoire de la représentation démocratique en France, Paris, NRF Gallimard.

Rosanvallon, P., 2000, La démocratie inachevée, Histoire de la souveraineté du peuple en France, Paris, NRF Gallimard.

Rosanvallon, P., 2003, Pour une histoire conceptuelle du politique, Paris, Seuil.

Zémor, P., 2003, Pour un meilleur débat public, Paris, Presses de Sciences Po.

\section{NOTES}

1. Un exemple parmi beaucoup d'autres est significatif : l'impuissance du discours de l'Eglise catholique et notamment du Pape dans le domaine de la contraception ou de l'avortement cohabite avec une puissance médiatique qui attire les foule, notamment de jeunes, partout où Jean-Paul II se rend; chacun trouve en lui ce qui lui plaît et laisse le reste.

2. Cf., pour une analyse approfondie cf. Debray, R., (1991 et 2001).

3. Wolton, D., 1996, dans un article d'Etudes (n³842) cité par Hartereau, A., 2002.

4. La réintroduction d'espèces sauvages présente de nombreuses vertus et sans doute quelques inconvénients qu'il serait trop long de développer ici. Disons simplement qu'il s'agit à la fois d'un symbole, d'un signe politique et d'un indicateur que certains dégâts du mal-développement peuvent être réparés.

5. Le conseil économique et social (ordonnance du 29 décembre 1958), les comités économiques et sociaux régionaux (loi du 2 février 1985), la commission locale d'information et de surveillance en matière de déchet (loi du 15 juillet 1976), les comités consultatifs d'habitants, les commissions consultatives pour les services publics locaux, etc.

6. Le concept de développement durable peut être considéré, étant données les conditions de son apparition et la vitesse fulgurante avec laquelle il s'est répandu sur la planète (avec certes, des effets encore peut visibles), comme le premier concept de l'ère mondialisée. 
7. On fait souvent remonter les prémices du développement durable aux travaux du « Club de Rame » qui, en 1972, préconisait la croissance zéro afin d'éviter l'épuisement des ressources. Outre que cette proposition a pu passer pour celle de riches qui auraient souhaité imposer aux pauvres de rester dans leur sous-développement, elle avait surtout la caractéristique négative de tuer tout espoir d'innovation, de recherche, de nouvelles voies..

8. Loi n99-533 du 25 juin 1999 d'orientation pour l'aménagement et le développement durable du territoire, article 25. L'article 26 atténue la portée du conseil de développement des agglomérations puisque celui-ci n'est que «consulté».

9. Cet exemple est bien réel: il s'est déroulé dans une assemblée plénière d'un conseil de développement au cours de laquelle le débat, visiblement ancien, sur la question de l'organisation des transports collectifs dans une zone rurale, ronronnait tranquillement entre " spécialistes »; jusqu'au moment où la présidente d'une association culturelle a pris la parole « en tant que mère de famille ». Cela a radicalement transformé la manière de "voir» cette question du transport et devrait déboucher sur des solutions jusque là insoupçonnées.

10. On limite volontairement le propos au niveau local d'une part parce que rien ne prouve que la question démocratique se pose, en pratique, de la même manière à tous les échelons territoriaux et d'autre part parce qu'en réalité, c'est la seule échelle à laquelle on constate aujourd'hui de véritables innovations malgré l'existence de la commission nationale du débat public et les expérimentations telles que les conférences de consensus. 11. Nous étudions cette question dans une étude à paraître, cf. Rayssac, G.L. et alii, à paraître

12. On part ici du principe, empirique, mais maintes fois vérifiées, qu'un dissensus clairement établi (par expression claire et reconnue des positions divergentes ou antagonistes) est plus productif, en termes de mobilisation pour l'action, qu'un faux consensus. De fait, cette méthode aboutit à des consensus positifs et des consensus sur les dissensus.

13. On appelle ici puissance administrative l'ensemble des services qui exécutent les décisions des élus, qu'il s'agisse de la fonction publique ou des « agences » para-publiques qui peuvent avoir, par ailleurs, un statut privé (comme nombre d'agences de développement) ou encore les organismes consulaires.

\section{RÉSUMÉS}

Le désenchantement démocratique qui se confirme régulièrement s'explique en partie par la désagrégation de l'espace public de délibération habermassien. Le développement durable offre une chance de redonner du sens et du contenu au débat politique. Parmi les différentes formules de structures de concertation proposées par le corpus législatif, le conseil de développement peut être un lieu offrant des caractéristiques encourageantes pour revivifier la participation des citoyens au débat démocratique, à condition que son fonctionnement prévoit une réelle procédure d'animation du débat public. Si ces deux conditions sont réunies (un lieu, une 
procédure), alors le conseil de développement peut être un outil de renouvellement de la gouvernance des territoires.

INDEX

Mots-clés : conseil de développement

\section{AUTEUR}

\section{GILLES-LAURENT RAYSSAC}

Gilles-Laurent Rayssac est Directeur de la Mission « Gouvernance et Développement Durable », Bernard Brunhes Consultants. A 41 ans, il est consultant après avoir été au début des années quatre-vingt secrétaire confédéral à la CFDT. Il travaille principalement dans le champ du développement territorial et des politiques d'emploi. Il a dirigé plusieurs importantes évaluations de politiques publiques dans ces deux domaines. Il expérimente, depuis quelques années, diverses méthodes d'animation de débat public, principalement dans le champ territorial.gilles-laurent.rayssac@brunhes.com 